their parental cells and tissues, and could serve as markers of pathology. Particularly in SLE, MPs are potential biomarkers and triggers of autoimmunity.Recent studies have demonstrated increased of plasmatic EMPs in patients with SLE active disease and their reduction after treatment.

The aim of this study was to investigate levels of EMPs in a cohort of SLE patients with and without renal involvement compared to healthy controls.

MPs were isolated from plasma and urine and characterised by flow cytometry using AnnessinV (a probe that binds to the exposed phosphatidilserine - PS)and antibodies against surface markers endothelial cells(CD31 +CD41-).

Sixty SLE patients and 29HC were studied. Twenty-eight patients had renal involvement.

The total number of plasmatic MPs was lower in SLE patients than $\mathrm{HC}(\mathrm{p}=0.001)$.

In contrast there was no significant difference EMPs between the two groups. When the patients were divided according to renal involvement, the patients with active-LN(A$\mathrm{LN})$ showed lower plasmatic EMPs in comparison to inactive $\mathrm{LN}(\mathrm{I}-\mathrm{LN}) \quad(\mathrm{p}=0.01)$, while the patients with I-LN had higher EMPs than $\mathrm{HC}(\mathrm{p}=0.002)$. There was no significant difference of total urinaryMPs between SLE patients and HC. UrinaryEMPs were higher in SLE and in LN patients than HC.

The results of the present study show increased EMPs in patients with LN in remission. Circulating-EMPs have been considered as a potential biomarker of endothelial activation and damage in several autoimmune disorders, and higher EMP have been detected in patients with vasculitis and associated with disease activation. According to our results, plasmatic EMPs are higher in inactive-LN patients than in HC. These results may suggest a potential role of EMP as a biomarker of $\mathrm{LN}$.

\section{PS2:25 ANALYSIS OF THE POLYAMINE METABOLOME IN THE PLASMA OF PATIENTS WITH SYSTEMIC LUPUS ERYTHEMATOSUS AND FEVER}

C Suh, W Baek, S Lee, H Kim, J Jung. Ajou University School of Medicine, Suwon, South Korea

\subsection{6/upus-2018-abstract.73}

Introduction Systemic lupus erythematosus (SLE) is a systemic autoimmune disease with various clinical manifestations and serologic markers. In this study, we analysed nine polyamine profiles of plasma from patients with SLE and healthy controls, and the relationship between the polyamine profiles and disease activity.

Methods The alterations of the polyamine metabolome in the plasma of 44 patients with SLE and fever were investigated using gas chromatography mass spectrometry in selected ion monitoring mode using N-ethoxycarbonyl/N-pentafluoropropionyl derivatives, and compared with those of 43 healthy controls.

Results Patients with SLE and healthy controls showed differences in five of nine polyamine metabolome profiles. Among five polyamines changed levels, four polyamines, namely N1acetylcadaverine, spermidine, N1-acetylspermidine, and spermine, were dramatically decreased. However, the level of cadaverine was increased in patients with SLE. In the partial correlation with polyamine profiles and disease activity markers of SLE, several disease activity markers and nutritional markers were correlated with cadaverine, spermidine, and N8-acetylspermidine.

Conclusion Thus, our results provide a comprehensive understanding of relationship between that polyamine metabolomes and disease activity markers in patients with SLE and fever.

\section{PS2:26 SLE PATIENTS WITH SECONDARY SJÖGREN'S SYNDROME ARE CHARACTERISED BY TYPICAL AUTOANTIBODIES AND A PRO-INFLAMMATORY STATE}

${ }^{1} \mathrm{M}$ Kvarnström, ${ }^{2} \mathrm{G}$ Ruacho, ${ }^{1} \mathrm{~J}$ Gustafsson, ${ }^{1} \mathrm{~A}$ Zickert, ${ }^{1} \mathrm{~V}$ Oke, ${ }^{3} \mathrm{~J}$ Rönnelid, ${ }^{4} \mathrm{~K}$ Elvin, ${ }^{1}$ I Gunnarsson, 'E Svenungsson. 'Unit of Rheumatology, Department of Medicine Solna, Karolinska Institutet, Karolinska University Hospital, Stockholm, Sweden; ${ }^{2}$ Centre for Clinical Research Sörmland, Uppsala University, Uppsala, Sweden; ${ }^{3}$ Department of Immunology, Genetics and Pathology, Uppsala University, Uppsala, Sweden; ${ }^{4}$ Unit of Clinical Immunology, Department of Clinical Immunology and Transfusion Medicine, Karolinska Institutet, Karolin, Stockholm, Sweden

\subsection{6/lupus-2018-abstract.74}

Background Sjögren's syndrome occurs in isolation (primary Sjögren's syndrome, pSS), but it is also often secondary (sSS) to, and sometimes difficult to delineate from systemic lupus erythematosus (SLE). Consequently there is a need to investigate similarities and differences between SLE patients with (SLE-sSS) and without sSS (SLE-noSS).

Objective To investigate the occurrence of sSS in a large cohort of SLE patients and to explore clinical and laboratory characteristics associated with SLE-sSS as compared to SLEnoSS and controls.

Methods We included 504 consecutive SLE patients and 322 population controls, matched for age and gender. All patients fulfilled the 1982 revised ACR criteria for SLE. SLE-sSS was defined according to the American-European consensus criteria (AECC). Subjective and objective quantifications of sicca symptoms were recorded. All underwent a thorough clinical investigation. SLE-associated autoantibodies, (ANA screening by BioPlex 2200 system, Bio-Rad) and Rheumatoid factor (Rf, Phadia Immunocap 250) were determined, Routine laboratory workup and a panel of cytokines (MSD 30-plex cytokine assays, performed on samples from 433 consecutive SLE patients and 319 controls) were measured.

Results SLE-sSS, occurred in 23\% of the SLE patients. Compared to SLE-noSS the SLE-sSS group was older, both at inclusion (55 vs 43 years, $\mathrm{p}<0.0001)$ and at disease onset $(40$ vs 32 yrs $\mathrm{p}<0.0001$ ), and more enriched in females (96 vs $83 \%, \mathrm{p}=0.0007)$, Leucopenia (57 vs $45 \%, \mathrm{p}=0.02)$ and peripheral neuropathy ( 15 vs $7 \%, \mathrm{p}=0.01)$ were more common and nephritis less common ( 32 vs $43 \%, p=0.03$ ). Higher levels of total IgG, positivity for anti-SSA/Ro52, anti-SSA/ Ro60, anti-SSB antibodies, Rf IgM and Rf IgA characterised the SLE-sSS group. 19/20 levels of detected cytokines were higher in SLE than in controls. 6/20 cytokines (TNF-a, IL-6, MCP-4, MIP-1 $\beta$, IL12/IL-23p40 and IP-10) were upregulated in SLE-sSS vs SLE-noSS (see table for figures).

Conclusion Frequency of SLE-sSS increases with age and affects roughly $1 / 4$ of SLE patients. Nephritis was less common while leucopenia and peripheral neuropathy were more common. We report higher levels of six pro-inflammatory. These findings demonstrate that, though often regarded as a milder version of SLE, patients with SLE-sSS are characterised by a state of chronic systemic inflammation. 
Abstract PS2:26 Table 1 Immunoglobulins autoantibodies and pro-inflammatory cytokines in SLE-SS, SLE-noSS and population controls

\begin{tabular}{|c|c|c|c|c|}
\hline & $\begin{array}{c}\text { Controls } \\
N=322 \\
\text { median (IQR) or } \\
N(\%)\end{array}$ & $\begin{array}{c}\text { SLE-SS } \\
N=117 \\
\text { median (IQR) or } \\
N(\%)\end{array}$ & $\begin{array}{c}\text { SLE-noSS } \\
N=387 \\
\text { median (IQR) or } \\
N(\%)\end{array}$ & $\begin{array}{c}\text { p-value } \\
\text { SLE-SS } \\
\text { vs. } \\
\text { SLE-noSS }\end{array}$ \\
\hline IgA total $\mathrm{g} / \mathrm{L}$ & $2.1(1.5 \cdot 2.8)$ & $2.9(1.8-4.3)$ & $2.7(1.9 \cdot 3.6)$ & 0.38 \\
\hline IgG total $g / \mathrm{L}$ & $10.9(9.5-12.2)$ & $14.5(10.4-18.3)$ & $12.4(9.8-15.8)$ & 0.009 \\
\hline IgM total g/L & $1.1(0.8-1.6)$ & $1.0(0.5 \cdot 1.6)$ & $0.9(0.6 \cdot 1.5)$ & 0.89 \\
\hline $\begin{array}{l}\text { anti-dsDNA \% positive } \\
(+)\end{array}$ & $5(1.6)$ & $36(31.3)$ & $154(41)$ & 0.06 \\
\hline anti-Ro52 \% + & $3(0.9)$ & $56(47.9)$ & $84(21.8)$ & $<0.0001$ \\
\hline anti-Ro60 \% + & $5(1.6)$ & 69(59) & $137(35.9)$ & $<0.0001$ \\
\hline anti-La/SSB \% + & $10(3.1)$ & $44(37.6)$ & $69(18)$ & $<0.0001$ \\
\hline anti-Sm \% + & $I(0.3)$ & $19(16.2)$ & $75(19.5)$ & 0.42 \\
\hline anti-RNP $68 \%+$ & $0(0)$ & $11(9.4)$ & $40(10.4)$ & 0.74 \\
\hline Rf IgG \%+ & $10 / 261(3.8)$ & $17 / 80(21.2)$ & $35 / 259(13.5)$ & 0.09 \\
\hline Rf IgM \% + & $14 / 283(4.9)$ & $32 / 83(38.6)$ & $56 / 281(19.9)$ & 0.0005 \\
\hline Rf IgA \%+ & $12 / 282(12.4)$ & $34 / 74(45.9)$ & $75 / 267(28.0)$ & 0.004 \\
\hline TNF- $\alpha \mathrm{pg} / \mathrm{mL}$ & $2.3(2.0-2.8)$ & $4.9(3.6-7.1)$ & $4.4(3.0 \cdot 6.0)$ & 0.008 \\
\hline IL-6 pg/mL & $0.5(0.4-0.7)$ & $1.5(0.8 \cdot 3.0)$ & $1.1(0.6 \cdot 2.0)$ & 0.009 \\
\hline MCP-4 pg/mL & $55.8(40.8-80.5)$ & $94.9(66.9 \cdot 131.3)$ & $74.7(52.4-120.0)$ & 0.019 \\
\hline MIP-1 $\beta \mathrm{pg} / \mathrm{mL}$ & $43.7(33.4-56.4)$ & $81.1((54.8-123.6)$ & $68.9(50.3-105.1)$ & 0.021 \\
\hline IL12/IL-23p40 pg/mL & $131.2(99.8-179.5)$ & $211.3(141.4-363.8)$ & $177.1(119.6274 .5)$ & 0.032 \\
\hline IP-10 pg/mL & $351.9(259.2-476.4)$ & $808(536.1911)$ & $726(440-1471)$ & 0.036 \\
\hline
\end{tabular}

\section{PS2:27 ANTIBODIES TO CARBAMYLATED VIMENTIN IN PATIENTS WITH SYSTEMIC LUPUS ERYTHEMATOSUS ARE ASSOCIATED WITH RENAL INVOLVENMENT}

FR Spinelli, T Colasanti, S Truglia, A Pecani, F Ceccarelli, F Miranda, E Moscarelli, R Mancini, C Perricone, C Alessandri, G Valesini, F Conti. Sapienza Università di Roma, Dipartimento di Medicina Interna e Specialità Mediche, Reumatologia, Rome, Italy

10.1136/lupus-2018-abstract.75

Vimentin is a cytoskeletal protein expressed by mesenchymal cells, including endothelial and renal tubular cells. Antibodies to vimentin were described in $10 \%-53 \%$ of patients with Systemic Lupus Erythematosus (SLE). Vimentin has been proposed as a target of the in situ immune response in lupus nephritis. Post-translational modifications increase the immunogenicity of vimentin, as demonstrated by the detection of antimodified-vimentin antibodies in rheumatoid arthritis. Carbamylation is a non-enzymatic post-translational modification (addition of a cyanate group on lysine and arginine residues), which has been linked to NETosis. The role of carbamylated vimentin (Car-Vim) as an antigenic target in SLE has not been evaluated yet.

Aim of the study was to assess the prevalence of anti-CarVIm and to investigate any association with clinical and serological features in SLE patients.

We enrolled SLE diagnosed according to 1997 ACR criteria. Clinical features, autoantibodies profile and disease activity according to SLEDAI $2 \mathrm{~K}$ - were collected. Patients' sera were tested for anti- Car-Vim by a home-made enzyme-linked immunoassay. Data were expressed as mean \pm standard deviation or median (interquartile range) when appropriate. Mann-Whitney and Chi square test were applied to investigate differences in anti- carbamylated vimentin prevalence and serum levels. P value $<0.05$ was considered statistically significant.

We enrolled 109 SLE patients (102F:7M, mean age 39.4 \pm 12.6 years, mean disease duration $10.5 \pm 9.5$ years, mean SLEDAI $2 \mathrm{~K} 5 \pm 5.5$ ). Table 1 summarises the main clinical and serological features. Overall, 30/109 patients (27.5\%) were positive for anti-Car-Vim. The prevalence of anti-Car-Vim was significantly higher in patients with lupus nephritis (18/44) compared to those without $(12 / 66)(41.8 \%$ vs $18.2 \%$, $\mathrm{p}=0.006)$; moreover, anti-Car-Vim serum levels were significantly higher in patients with lupus nephritis [2561 (1783) OD] compared to those without [1970 (1123) OD; $\mathrm{p}=0.0178]$. No difference was found in prevalence or titre of anti-Car-Vim in presence/absence of other clinical or serological manifestations. No correlation between anti-Car-Vim serum levels and SLEDAI $2 \mathrm{~K}$ was found.

Higher prevalence and serum levels of anti-carbamylated vimentin antibodies in patients with lupus nephritis confirm the role of vimentin as a target of the immune response in glomerulonephritis and suggest their possible role as a biomarker of kidney involvement in SLE.

Abstract PS2:27 Table 1 Clinical and serological feature of the patients at the time of enrolment

\begin{tabular}{lc}
\hline Clinica/seroligical feature & $\mathbf{N}(\%)$ \\
\hline Artrhritis & $15(13.8)$ \\
Skin involvement & $16(14.7)$ \\
Lupus nephritis & $43(39.4)$ \\
CNS lupus & $7(6.4)$ \\
Serositis & $3(2.7)$ \\
Hematological disorders & $21(19.3)$ \\
Anti-dsDNA+ & $39 / 74(52.7)$ \\
Low complement levels & $35 / 61(57.4)$ \\
\hline
\end{tabular}

\section{PS2:28 AUTOANTIBODY PROFILING IN PROSTVAC AND IPILIMUMAB TREATED PROSTATE CANCER PATIENTS REVEALS POTENTIAL BIOMARKERS OF IMMUNE- RELATED ADVERSE EVENTS}

${ }^{1} \mathrm{P}$ Budde, ${ }^{2} \mathrm{~J}$ Marte, ${ }^{1} \mathrm{H}-\mathrm{D}$ Zucht, 'S Bhandari, 'M Tuschen, ${ }^{1} \mathrm{P}$ Schulz-Knappe, ${ }^{2} \mathrm{~J}$ Gulley, ${ }^{3} \mathrm{C}$ Heery, ${ }^{2} \mathrm{R}$ Madan, ${ }^{2} \mathrm{~J}$ Schlom. ${ }^{1}$ Protagen AG, Dortmund, Germany, Dortmund, Germany; ${ }^{2}$ National Cancer Institute, National Institutes of Health, Bethesda, MD, USA; ${ }^{3}$ Bavarian Nordic, Inc., Morrisville, NC, USA

\subsection{6/lupus-2018-abstract.76}

Purpose Autoantibodies (AAB) targeting self-antigens can be found in two clinically and immunologically opposing diseases, autoimmune diseases and cancer. While in autoimmune diseases, the immune system is hyperactivated against self-antigens, many tumours suppress the anti-tumour immune response. The therapeutic cancer vaccines PSA-Tricom (Prostvac) is designed to generate an antigen-specific tumour response in metastatic castration-resistant prostate cancer (mCRPC), which is in phase 3 testing. To further augment the immune response, combination therapies of Prostvac with ipilimumab are currently tested in clinical studies. Ipilimumab is an antibody that blocks the immune checkpoint molecule cytotoxic T-lymphocyte-associated antigen 4 (CTLA-4). However, 